\title{
A phase II study of daily carboplatin plus irradiation followed by durvalumab for stage III non-small cell lung cancer patients with PS 2 up to 74 years old and patients with PS 0 or 1 from 75 years: NEJ039A (trial in progress)
}

Kyoichi Kaira ${ }^{1 *}$, Atsuto Mouri ${ }^{1}$, Shingo Kato ${ }^{2}$, Kenichi Yoshimura ${ }^{3}$, Hiroshi Kagamu ${ }^{1}$ and Kunihiko Kobayashi ${ }^{1}$

\begin{abstract}
Background: Durvalumab is a standard drug used during maintenance therapy after chemoradiotherapy in patients with locally advanced non-small cell lung cancer (LA-NSCLC). However, little is known about the clinical benefits of durvalumab after chemoradiotherapy in patients with LA-NSCLC with a performance status (PS) of 2 and/or aged $>75$ years. As daily carboplatin plus concurrent thoracic radiotherapy is recommended for elderly patients according to guideline, the current phase II study aims to investigate the effect of daily carboplatin plus radiotherapy followed by durvalumab for patients with stage III NSCLC who have a PS of 2 and/or are older.
\end{abstract}

Methods: Daily carboplatin plus radiotherapy followed by durvalumab is performed for the patients with stage III NSCL $C$ who have a PS of 2 and/or are older. This is a trial in progress manuscript.

Study treatment: Daily, intravenous, low-dose carboplatin (30 mg/ $\mathrm{m}^{2}$ in a $30-\mathrm{min}$ infusion) is administered to patients $1 \mathrm{~h}$ before radiotherapy for the first 20 fractions. Radiotherapy for all patients consisted of $60 \mathrm{~Gy}$ administered as 30 fractions over 6 weeks. Durvalumab at a dose of $10 \mathrm{mg} / \mathrm{kg} / \mathrm{body}$ is intravenously administered every 2 weeks for up to 12 months after chemoradiotherapy.

Exploratory assessment: In the future, an exploratory investigation will be performed to determine whether the combined assessment of T-cell markers, PD-L1 expression, and tumor mutation burden could predict the outcomes of the regimen.

Discussion: The results of our study will exhibit the efficacy and tolerability of durvalumab as maintenance therapy after daily carboplatin plus radiotherapy.

(Continued on next page)

\footnotetext{
*Correspondence: kkaira1970@yahoo.co.jp

'Department of Respiratory Medicine, Comprehensive Cancer Center,

International Medical Center, Saitama Medical University, 1397-1 Yamane,

Hidaka-City, Saitama 350-1298, Japan

Full list of author information is available at the end of the article
}

(c) The Author(s). 2020 Open Access This article is licensed under a Creative Commons Attribution 4.0 International License, which permits use, sharing, adaptation, distribution and reproduction in any medium or format, as long as you give appropriate credit to the original author(s) and the source, provide a link to the Creative Commons licence, and indicate if changes were made. The images or other third party material in this article are included in the article's Creative Commons licence, unless indicated otherwise in a credit line to the material. If material is not included in the article's Creative Commons licence and your intended use is not permitted by statutory regulation or exceeds the permitted use, you will need to obtain permission directly from the copyright holder. To view a copy of this licence, visit http://creativecommons.org/licenses/by/4.0/ The Creative Commons Public Domain Dedication waiver (http://creativecommons.org/publicdomain/zero/1.0/) applies to the data made available in this article, unless otherwise stated in a credit line to the data. 


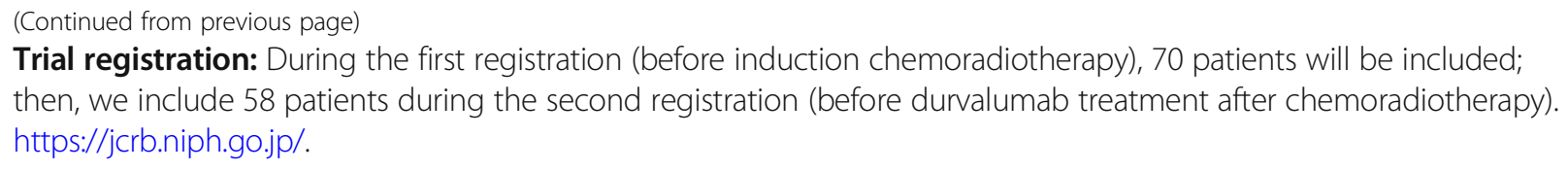

Primary endpoint: The primary endpoint of the current study is the 12-month progression-free survival (PFS) rate after the initiation of durvalumab.

Secondary endpoints: The secondary endpoints are the feasibility, objective response, PFS, overall survival, and adverse events.

Keywords: Durvalumab, Chemoradiotherapy, Locally advanced non-small cell lung cancer, Elderly, PS 2, Carboplatin, Immune checkpoint inhibitor, PD-L1 antibody

\section{Background}

Chemoradiotherapy is a standard treatment for patients with locally advanced stage III non-small cell lung cancer (NSCLC). Combination regimens used in daily practice include platinum derivatives plus third-generation agents such as cisplatin plus docetaxel [1] and carboplatin plus paclitaxel [2]. However, chemoradiotherapy has not been widely applicable for patients with locally advanced NSCLC (LA-NSCLC). Accordingly, most patients with a performance status (PS) of 0 to 1 and aged less than 70 years receive chemoradiotherapy [1-5]. The remaining patients are treated with radiotherapy alone without chemotherapy and immune checkpoint inhibitors (ICIs). Therefore, the outcomes of these patients are poor, with a 5 -year survival rate of $<10 \%$ compared to the 5 -year survival rate of approximately $15 \%$ observed when patients receive chemoradiotherapy $[6,7]$.

For patients with a poor PS and elderly patients, less toxic therapeutic regimens should be considered in combination with radiotherapy. Carboplatin is a platinum compound that crosslinks with DNA and is associated with a lower incidence of nephrotoxicity, neurotoxicity, nausea, and vomiting than cisplatin [8]. Moreover, carboplatin and cisplatin have similar radiosensitizing properties $[9,10]$. A total radiation dose of 60 Gy in 2-Gy fractions with daily carboplatin $\left(30 \mathrm{mg} / \mathrm{m}^{2}\right.$ in a $30-\mathrm{min}$ infusion), when administered $1 \mathrm{~h}$ before radiotherapy for the first 20 fractions, was found to be effective for prolonging overall survival (OS) with acceptable tolerability [11]. Therefore, per the treatment guidelines in Japan, daily carboplatin plus thoracic concurrent radiotherapy is the recommended treatment.

Carboplatin also has the ability to change the immune status of patients [12]. In fact, part of the antitumor effects of platinum drugs occurs through modulation of the immune system. These immunogenic effects include modulation of STAT signaling; induction of an immunogenic type of cancer cell death through exposure of calreticulin and release of ATP and high-mobility group protein box-1 (HMGB-1); and enhancement of the effector immune response through modulation of programmed death receptor 1-ligand (PD-L1) and mannose-6-phosphate receptor expression. Accordingly, at least part of the antitumor effect of platinum chemotherapeutics may be due to immune-potentiating mechanisms.

Durvalumab is high-affinity, human IgG1 monoclonal antibody that blocks PD-L1 from binding to programmed death 1 (PD-1) and CD80 [13]; a clinical trial proved the antitumor activity of durvalumab in patients with several advanced solid tumors such as NSCLC [14]. The PACIFIC study compared durvalumab to a placebo to determine the prognostic significance of maintenance therapy in patients with stage III NSCLC who received concurrent thoracic radiotherapy with platinum-based chemotherapy $[15,16]$. The PACIFIC study demonstrated that progression-free survival (PFS) was significantly longer after durvalumab (16.8 months) than after placebo (5.6 months; stratified hazard ratio for disease progression or death, $0.52 ; P<0.001)$. In addition, the 12 -month PFS rate was $55.9 \%$ (versus $35.3 \%$ for placebo). Accordingly, durvalumab after concurrent chemoradiotherapy is widely used for treating patients with locally advanced NSCLC; however, many patients who have a PS of 2 and/or are older are not treated using this combination, because the clinical evidence of this treatment is excluded from the PACIFIC study.

Currently, thoracic radiotherapy alone is the standard of care for elderly patients with LA-NSCLC in Japan, especially those aged $>75$ years. However, the combination of daily carboplatin plus concurrent thoracic radiotherapy might be chosen for elderly patients with a good PS and adequate tolerability. On the basis of the evidence of the PACIFIC study, it remains unclear whether a platinum-based regimen plus concurrent thoracic radiation followed by durvalumab results in significant survival prolongation for elderly patients aged $>75$ years when compared to chemoradiotherapy with daily carboplatin. Similarly, little is known about the clinical benefit of durvalumab after chemoradiotherapy for LA-NSCLC patients with a PS of 2.

Therefore, the current phase II study aims to investigate the survival benefit of daily carboplatin plus 
radiotherapy followed by durvalumab for patients with stage III NSCLC, including those who have a PS of 2 and/or are older.

\section{Methods / design}

\section{Study design and objective}

The current non-randomized, prospective, open-label phase II study aims to evaluate the efficacy of daily carboplatin plus concurrent thoracic radiation followed by durvalumab for patients with stage III NSCLC who have a PS of 2 and/or are older. The design and protocol of this study are shown in Fig. 1.

The primary endpoint of this study is the 12-month PFS rate from the initiation of durvalumab. The secondary endpoints are the feasibility, objective response, PFS, OS, and adverse events. As an exploratory analysis, a predictive biomarker for this regimen will be examined using T-cell markers such as CD62LlowCD4 $+\mathrm{T}$ cells and CD25 + Foxp $3+$ CD4+ in the peripheral blood, and considering the expression of PD-L1 within tumor cells and tumor mutation burden before treatment. All procedures will be performed in accordance with the ethical standards of the institutional and/or national research committee and with the 2013 Declaration of Helsinki and its later amendments or comparable ethical standards. Written informed consent is obtained from all participants in our study. This study has been registered in the Japan Registry of Clinical Trials (JRCT) (jRCTs031190070).

\section{Key eligibility criteria}

The inclusion and exclusion criteria during the first registration are listed in Table 1 . For administering durvalumab after chemoradiotherapy with daily carboplatin plus concurrent thoracic irradiation, the second registration will be performed according to the additional inclusion and exclusion criteria, as listed in Table 2.

\section{Treatment schedule}

Daily, intravenous, low-dose carboplatin $\left(30 \mathrm{mg} / \mathrm{m}^{2}\right.$ in a 30-min infusion) is administered $1 \mathrm{~h}$ before radiotherapy for the first 20 fractions. Planned radiotherapy of $60 \mathrm{~Gy}$ is administered as 30 fractions from 6 to 9 weeks. Radiotherapy is administered 5 days per week (i.e., Monday to Friday with the weekend off) in 2 Gy daily fractions via 6-18 MV X-rays. Both three-dimensional conformal radiotherapy and intensity-modulated radiotherapy are acceptable. Radiation doses are prescribed according to the planning target volume. Motion management is performed as required, and the internal target volume, clinical target volume, and planning target volume vary according to the motion management method used. The use of positron emission tomography (PET) or computed tomography $(\mathrm{CT})$ and four-dimensional $\mathrm{CT}$ for

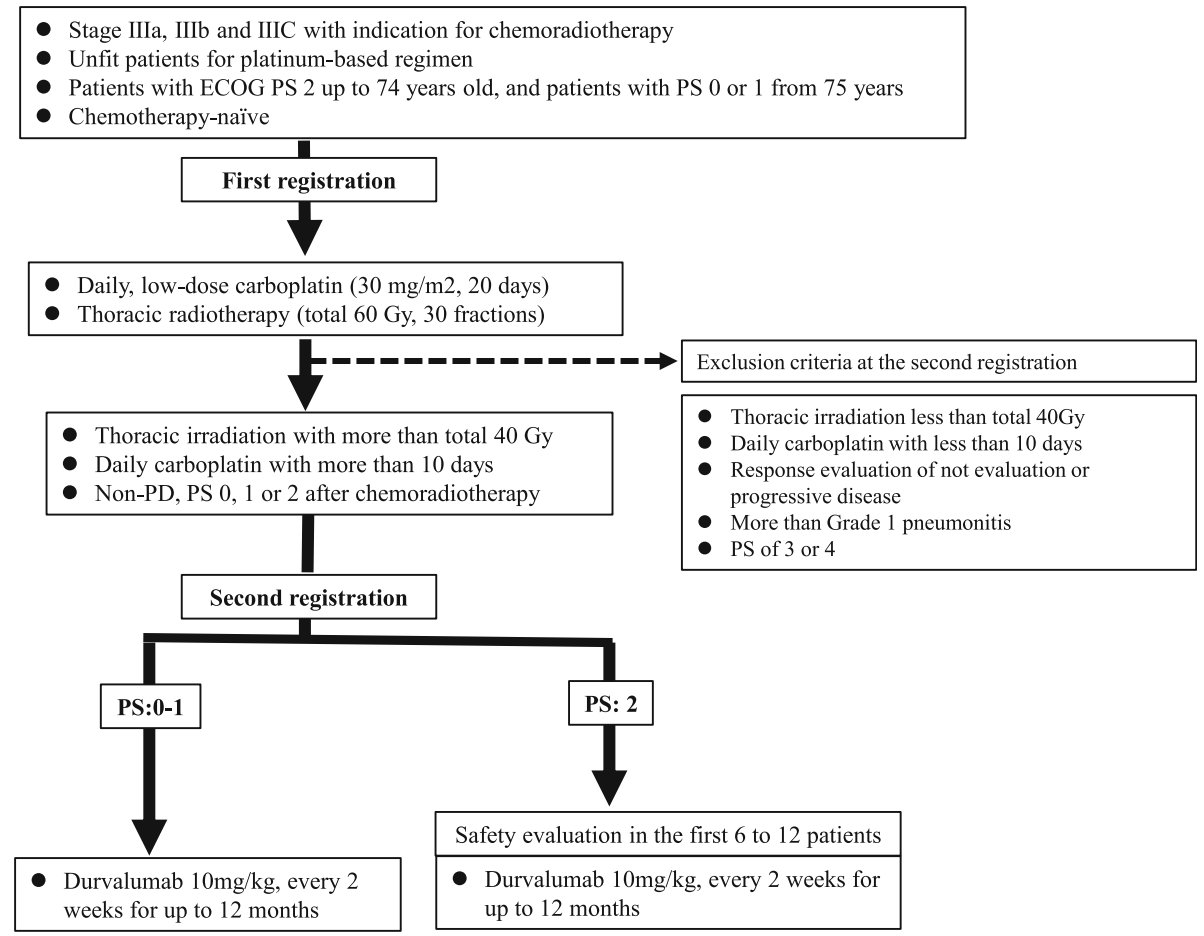

Fig. 1 Design and protocol of this study 
Table 1 Inclusion and exclusion criteria in the first registration

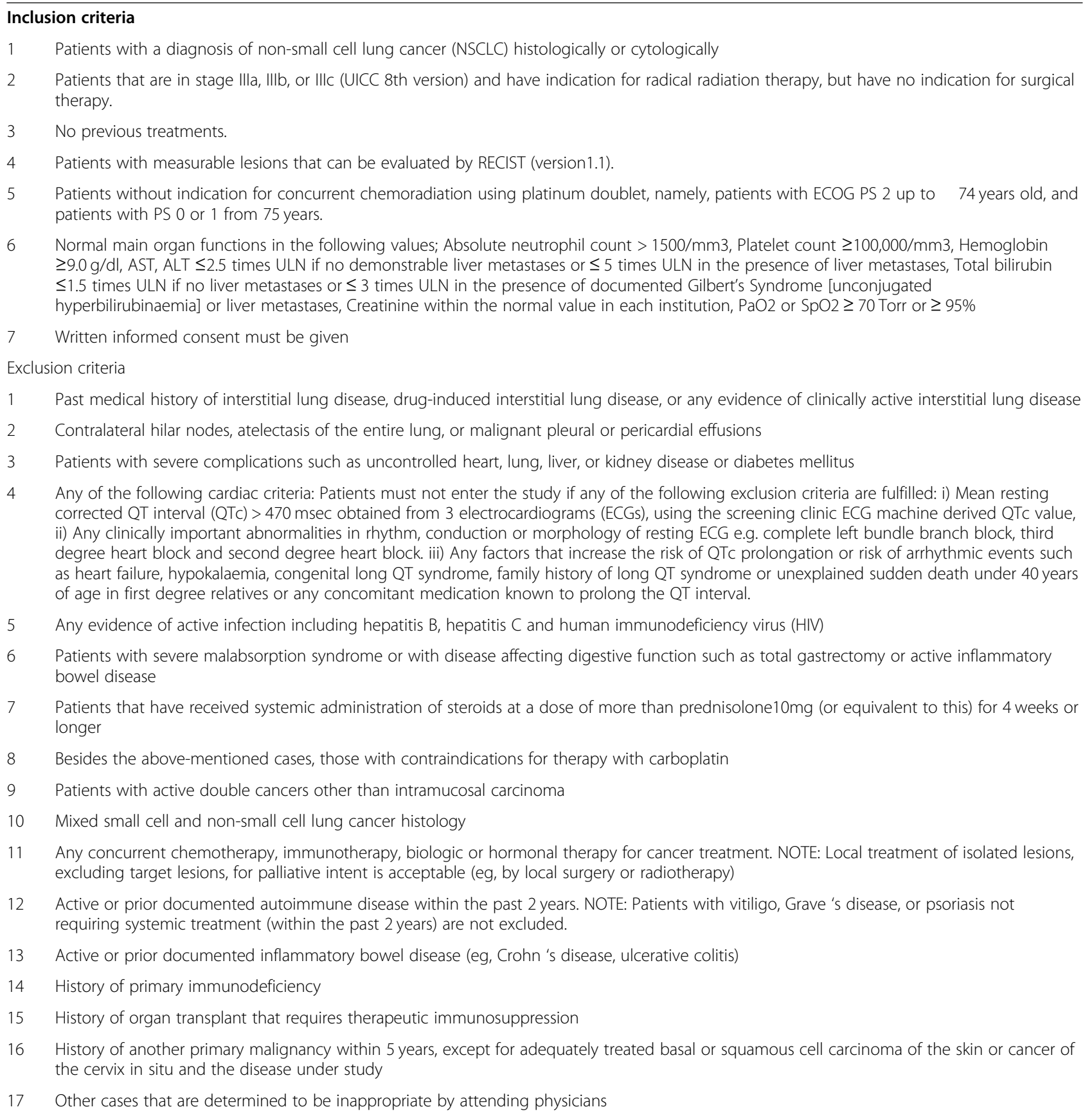

radiotherapy planning is encouraged. The gross tumor volume is defined as the primary tumor and any regionally involved nodes on CT (> $1 \mathrm{~cm}$ on the short axis) or pretreatment PET (standardized uptake value $>3$ ). The internal target volume is defined as the envelope that encompassed the gross tumor volume plus the ventilatory motion. The clinical target volume margins are 0.5$1.0 \mathrm{~cm}$ beyond the internal target volume. The planning target volume margins are $0.5-1.5 \mathrm{~cm}$ beyond the clinical target volume, depending on the use of fourdimensional CT for planning and image-guided radiotherapy for delivery. The V20 (the volume of the lung parenchyma that received $20 \mathrm{~Gy}$ or more) needs to be less than $30 \%$.

Durvalumab is administered 1 to 42 days after the patients receive chemoradiotherapy. Durvalumab is intravenously administered at a dose of $10 \mathrm{mg}$ per kilogram of body weight every 2 weeks for up to 12 months. 
Table 2 Additional inclusion and exclusion criteria in the second registration

Inclusion criteria
Completion of thoracic irradiation with more than total $40 \mathrm{~Gy}$
2 Daily carboplatin with more than 10 day
Non-PD and PS 0, 1 or 2 after chemoradiotherapy
3 Normal main organ functions in the following values; Absolute
4 neutrophil count $>1500 / m m 3$, Platelet count $\geq 100,000 / m m 3$,
Hemoglobin $\geq 9.0$ g/dl, AST, ALT $\leq 2.5$ times ULN if no
demonstrable liver metastases or $\leq 5$ times ULN in the presence of
liver metastases, Total bilirubin $\leq 1.5$ times ULN if no liver
metastases or $\leq 3$ times ULN in the presence of documented
Gilbert's Syndrome [unconjugated hyperbilirubinaemia] or liver
metastases, Creatinine within the normal value in each institution,
PaO2 or SpO2 $\geq 70$ Torr or $\geq 95 \%$

Exclusion criteria

Patients with locally advanced NSCLC who have progressed whilst

1 radiotherapy concurrent with daily carboplatin

Any unresolved toxicity CTCAE > Grade 2 from the prior

2 chemoradiation therapy

Patients with any grade pneumonitis from prior chemoradiation

3 therapy

Uncontrolled intercurrent illness including, but not limited to,

4 ongoing or active infection, symptomatic congestive heart failure, uncontrolled hypertension, unstable angina pectoris, cardiac arrhythmia, active peptic ulcer disease or gastritis, active bleeding diatheses including any patient known to have hepatitis B, hepatitis $C$ or human immunodeficiency virus (HIV), or psychiatric illness/social situations that would limit compliance with study requirements or compromise the ability of the patient to give written informed consent

Receipt of live attenuated vaccination within 30 days prior to study

5 entry or within 30 days of receiving Durvalumab

Recent major surgery within 4 weeks prior to entry into the study

6 (excluding the placement of vascular access) that would prevent administration of Durvalumab.

Abbreviation: $P D$ progressive disease; $P S$ performance status; $\mathrm{AST}_{\text {i }} \mathrm{ALT}$; $\mathrm{NSCL}$ $C$, non-small cell lung cancer; CTCAE,

\section{Statistical analysis}

The primary endpoint is to evaluate the progression free survival rate of 12 months from the initiation of durvalumab administration. The secondary endpoint is to investigate the time to failure, the rate of therapeutic completion, progression-free survival, 2-year survival rate, objective response rate, safety and exploratory biomarker assessment. In the PACIFIC study, the 12 -month PFS rate was $55.6 \%$ after chemoradiotherapy followed by durvalumab and $35.3 \%$ after chemoradiotherapy alone. In the JCOG0301 study, the 12-month PFS rate was approximately 30\% after radiotherapy with daily carboplatin [11]. According to the retrospective study conducted by Arslan et al. [17], the 1-year OS rate of patients with LA-NSCLC with a PS of 2 was approximately $50 \%$. This was lower than the 1-year OS rate for elderly patients with a good PS (70.8\%) observed in the JCOG0301 study. The degree of difference in OS between patients with a poor PS (included in the study by Arslan et al.) and elderly patients (included in the JCOG0301 study) is approximately $30 \%$, which could be applied to the difference in PFS between these populations. Therefore, we assumed that a 12-month PFS rate of $35 \%$ after radiotherapy with daily carboplatin followed by durvalumab in eligible patients would indicate potential clinical usefulness, whereas a rate of $20 \%$ would be the lower limit for indicating clinical usefulness. On the basis of this assumption, our study was designed to have a power of $80 \%$ and had an alpha level of 0.1 (two-sided). The target number of patients for this study is 53 . Therefore, we assess 58 patients considering the progressive disease (PD) rate after treatment with radiation with daily carboplatin and patients who dropped out. In the JCOG0301 study, the PD rate was $8 \%$, the rate of treatment-related death was $3 \%$, and the rate of Grade 3-4 adverse events was $6 \%$, so we assumed the dropout rate to be $20 \%$. During the first registration, 70 patients will be included; then, we analyze 58 patients included during the second registration. A minimum follow-up of 12 months is needed after the last patient starts treatment with durvalumab.

Safety evaluation:

We are also investigating the safety in the first 6 patients with a PS of 2.

Further enrollment of patients with a PS of 2 will be stopped until the safety is confirmed in these 6 patients according to the following criteria (Fig. 2):

- Patients will be considered to have dose limiting toxicities (DLTs) if durvalumab treatment is interrupted more than two times owing to $>$ Grade 2 pneumonitis or the occurrence of $>$ Grade 3 adverse events.

- The DLTs will be evaluated during the period of 8 weeks after the administration of durvalumab 4 times.

- If $<2$ patients show DLTs, durvalumab will be deemed to be safe, and the inclusion of more patients with a PS of 2 will be restarted.

- If 3 DLTs are observed in the first 6 patients, 6 more patients will be included; then, if fewer than 5 of the 12 patients experience a DLT, the enrollment of patients with a PS of 2 will be restarted. Of the 12 patients, if 6 or more experience a DLT, further enrollment of patients with a PS of 2 will be stopped.

\section{ParticipatXing institutions}

The participating institutions include 31 hospitals in Japan. 


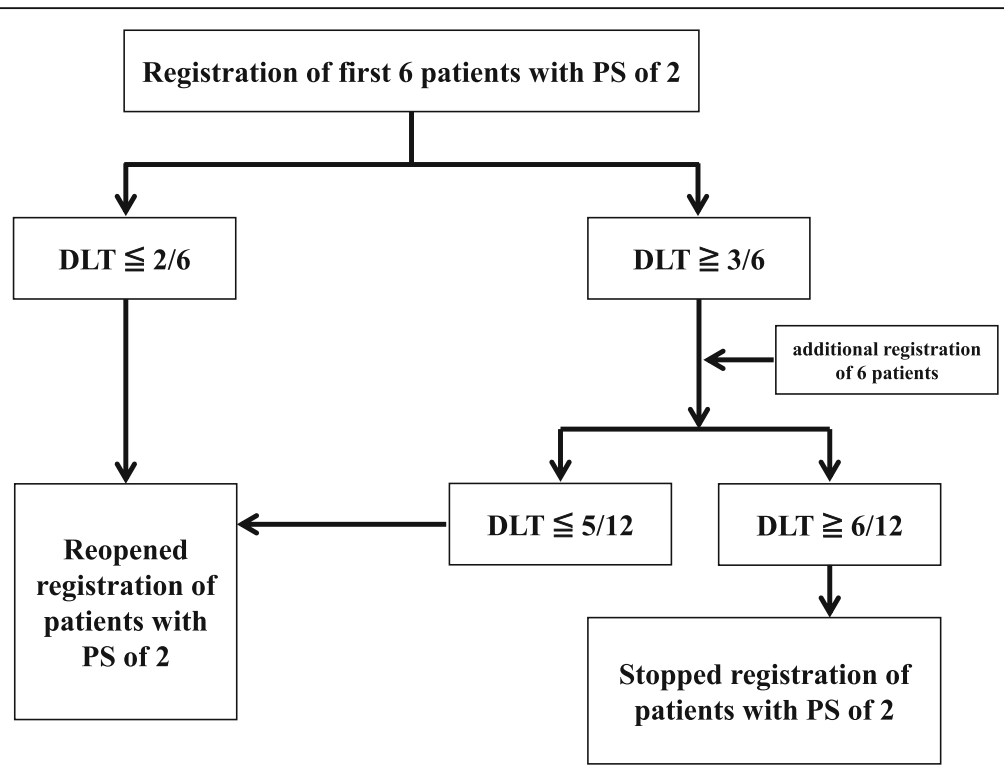

Fig. 2 Safety evaluation in 6 to 12 patients with PS of 2

\section{Discussion}

This is the first prospective study to evaluate the efficacy and feasibility of durvalumab as maintenance therapy after chemoradiotherapy for patients with unresectable stage III NSCLC who have a PS of 2 and/or are older (i.e., aged $>75$ years). In particular, it remains unclear whether durvalumab could display a significant clinical benefit in patients with a PS of 2 after chemoradiotherapy; therefore, the results of our study are expected to elucidate this clinical difficulty. The JCOG0301 study demonstrated that the 5-year survival rate was approximately $15 \%$, which was less than $20 \%$, observed in elderly patients with LA-NSCLC who received daily carboplatin plus concurrent radiotherapy [11]. In future, we expect that the 5-year survival rate will increase to > $20-30 \%$ after additional treatment with durvalumab, although our investigation is a phase II study to evaluate the 12-month PFS rate from the initiation of durvalumab. Till date, no prospective study has elucidated the clinical significance of chemoradiotherapy for patients with LA-NSCLC with a PS of 2. Although anti-PD-1/ PD-L1 antibodies result in a significant survival benefit for patients with advanced NSCLC with a good PS [18], the efficacy of ICIs is known to increase after radiotherapy [19]; therefore, a synergistic effect is expected for patients with a PS of 2. If mild toxic regimens, such as daily carboplatin-as used in the current study-are combined with concurrent thoracic radiotherapy, the regimen might be feasible for patients with a PS of 2, and sequential therapy with durvalumab will have the potential of improving the survival of such patients. Similarly, ICIs are feasible and effective for elderly patients with NSCLC (aged $\geq 75$ years) [18], and we believe that durvalumab as maintenance therapy after chemoradiotherapy is suitable for such patients.

As biomarkers, PD-L1 and the tumor mutation burden are broadly useful for predicting the efficacy of ICIs in patients with advanced NSCLC [19-21]. Recently, we reported that the increased level of CD62LlowCD4 $+\mathrm{T}$ cells in the peripheral blood could effectively predict the efficacy and survival of patients with advanced NSCLC who received anti-PD1 antibodies [22]. It remains unclear whether the value of CD62LlowCD4 $+\mathrm{T}$ cells is closely associated with the survival benefit of durvalumab after chemoradiotherapy; however, our exploratory investigation is necessary, as the results will help in the selection of patients who are sensitive to durvalumab.

\section{Conclusion}

The results of the NEJ039A study will exhibit the efficacy and tolerability of durvalumab as maintenance therapy after daily carboplatin plus radiotherapy, which is among the potential promising first-line treatment regimens for patients who have a PS of 2 and/or are older (aged $>75$ years). Moreover, the results of our exploratory investigation are expected to confirm whether the combined assessment of T-cell markers, PD-L1 expression, and tumor mutation burden could predict the outcomes of this regimen. 


\section{Abbreviations}

LA-NSCLC : Locally advanced non-small cell lung cancer; PS : Performance status; PFS : Progression-free survival; NSCLC : Non-small cell lung cancer; HMGB-1: Overall survival: OS; high-mobility group protein box-1; PD-

L1: Programmed death receptor 1-ligand; PD-1: Programmed death 1; JRCT : Japan Registry of Clinical Trials; PET : Positron emission tomography; CT

: Computed tomography; DLTs : Dose limiting toxicities

\section{Acknowledgments}

The authors thank the patients, their families, the coordinators, and NEJ039A study investigators. We especially appreciate Yuichi Takiguchi (Chiba University Hospital), Akinobu Yoshimura (Tokyo Medical University Hospital) and Hirohisa Yoshizawa (Niigata Medical Center) for their efforts as members of the independent data monitoring committee. This study is conducted with the dedicated support of NEJ staffs.

\section{Authors' contributions}

K kaira, AM, SK, HK and K kobayashi conducted study. K kaira, AM and K kobayashi wrote study protocol. K kaira and AM wrote the manuscript. All authors contributed to the review of the manuscript and all approved final draft for submission.

\section{Funding}

This study is funded by AstraZeneca Company. The study drug Durvalumab is not provided by AstraZeneca Company. The design, management, analysis, reporting and publication of the study are entirely independent of AstraZeneca Company.

\section{Availability of data and materials}

Not applicable.

\section{Ethics approval and consent to participate}

This study was an institutionally approved by Saitama Medical University. All procedures will be performed in accordance with the ethical standards of the institutional and/or national research committee and with the 2013 Declaration of Helsinki and its later amendments or comparable ethical standards. Written informed consent was obtained from all participants in our study. This study has been registered in the Japan Registry of Clinical Trials (JRCT) (jRCTs031190070).

\section{Consent for publication}

Not applicable.

\section{Competing interests}

K. Kaira has received research grants and a speaker honorarium from Ono Pharmaceutical Company, Boehringer Ingelheim, Chugai Pharmaceutical, Taiho Pharmaceutical, Eli Lilly Japan and AstraZeneca. AM has received a speaker honorarium from Eli Lilly, Taiho Pharmaceutical, Pfizer, Chugai Pharmaceutical and AstraZeneca. KY has received a speaker honorarium from AstraZeneca. HK has received research grants and a speaker honorarium from Ono Pharmaceutical Company, Bristol-Myers Company, Boehringer Ingelheim, MSD, Daiichi Sankyo Company, Chugai Pharmaceutical, Taiho Pharmaceutical, Merck Biopharma Company, Eli Lilly Japan and AstraZeneca. K. Kobayashi has received research grants and a speaker honorarium from Boehringer Ingelheim, AstraZeneca and Bristol-Myers Company. SK has no conflicts of interest.

\section{Author details}

'Department of Respiratory Medicine, Comprehensive Cancer Center, International Medical Center, Saitama Medical University, 1397-1 Yamane, Hidaka-City, Saitama 350-1298, Japan. ²Department of Radiation Oncology, Comprehensive Cancer Center, International Medical Center, Saitama Medical University, 1397-1 Yamane, Hidaka-City, Saitama 350-1298, Japan. ${ }^{3}$ Center for Integrated Medical Research, Hiroshima University Hospital, Hiroshima University, 1-2-3 Kasumi, Minami-ku, Hiroshima 732-8551, Japan.
Received: 3 April 2020 Accepted: 14 September 2020

Published online: 06 October 2020

\section{References}

1. Segawa Y, Kiura K, Takigawa N, Kamei H, Harita S, Hiraki S, Watanabe Y, Sugimoto K, Shibayama T, Yonei T, et al. Phase III trial comparing docetaxel and cisplatin combination chemotherapy with mitomycin, vindesine, and cisplatin combination chemotherapy with concurrent thoracic radiotherapy in locally advanced non-small-cell lung cancer: OLCSG 0007. J Clin Oncol. 2010;28(20):3299-306.

2. Bradley JD, Paulus R, Komaki R, Masters G, Blumenschein G, Schild S, Bogart J, Hu C, Forster K, Magliocco A, et al. Standard-dose versus high-dose conformal radiotherapy with concurrent and consolidation carboplatin plus paclitaxel with or without cetuximab for patients with stage IIIA or IIIB nonsmall-cell lung cancer (RTOG 0617): a randomised, two-by-two factorial phase 3 study. Lancet Oncol. 2015;16(2):187-99.

3. Albain KS, Crowley JJ, Turrisi AT 3rd, Gandara DR, Farrar WB, Clark JI, Beasley KR, Livingston RB. Concurrent cisplatin, etoposide, and chest radiotherapy in pathologic stage IIIB non-small-cell lung cancer: a southwest oncology group phase II study, SWOG 9019. J Clin Oncol. 2002;20(16):3454-60.

4. Curran WJ Jr, Paulus R, Langer CJ, Komaki R, Lee JS, Hauser S, Movsas B, Wasserman T, Rosenthal SA, Gore E, et al. Sequential vs. concurrent chemoradiation for stage III non-small cell lung cancer: randomized phase III trial RTOG 9410. J Natl Cancer Inst. 2011;103(1):1452-60.

5. Senan S, Brade A, Wang LH, Vansteenkiste J, Dakhil S, Biesma B, Martinez Aguillo M, Aerts J, Govindan R, Rubio-Viqueira B, et al. PROCLAIM: randomized phase III trial of Pemetrexed-Cisplatin or Etoposide-Cisplatin plus thoracic radiation therapy followed by consolidation chemotherapy in locally advanced nonsquamous non-small-cell lung Cancer. J Clin Oncol. 2016:34(9):953-62.

6. Aupérin A, Le Péchoux C, Rolland E, Curran WJ, Furuse K, Fournel P, Belderbos J, Clamon G, Ulutin HC, Paulus R, et al. Meta-analysis of concomitant versus sequential radiochemotherapy in locally advanced nonsmall-cell lung cancer. J Clin Oncol. 2010;28(13):2181-90.

7. Ahn JS, Ahn YC, Kim JH, Lee CG, Cho EK, Lee KC, Chen M, Kim DW, Kim HK, Min YJ, et al. Multinational randomized phase III trial with or without consolidation chemotherapy using Docetaxel and Cisplatin after concurrent Chemoradiation in inoperable stage III non-small-cell lung Cancer: KCSGLU05-04. J Clin Oncol. 2015;33(24):2660-6.

8. Calvert AH, Harland SJ, Newell DR, Siddik ZH, Jones AC, McElwain TJ, Raju S, Wiltshaw E, Smith IE, Baker JM, et al. Early clinical studies with cis-diammine1,1-cyclobutane dicarboxylate platinum II. Cancer Chemother Pharmacol. 1982;9(3):140-7.

9. Knox RJ, Friedlos F, Lydall DA, Roberts JJ. Mechanism of cytotoxicity of anticancer platinum drugs: evidence that cis-diamminedichloroplatinum (II) and cis-diammine-(1,1-cyclobutanedicarboxylato) platinum (II) differ only in the kinetics of their interaction with DNA. Cancer Res. 1986;46(4 Pt2):19729.

10. O'Hara JA, Douple EB, Richmond RC. Enhancement of radiation-induced cell kill by platinum complexes (carboplatin and iproplatin) in V79 cells. Int J Radiat Oncol Biol Phys. 1986;12(8):1419-22.

11. Atagi S, Kawahara M, Yokoyama A, Okamoto H, Yamamoto N, Ohe Y, Sawa T, Ishikura S, Shibata T, Fukuda H, et al. Thoracic radiotherapy with or without daily low-dose carboplatin in elderly patients with non-small-cell lung cancer: a randomised, controlled, phase 3 trial by the Japan clinical oncology group (JCOG0301). Lancet Oncol. 2012;13(7):671-8.

12. Hato SV, Khong A, de Vries IJ, Lesterhuis WJ. Molecular pathways: the immunogenic effects of platinum-based chemotherapeutics. Clin Cancer Res. 2014;20(11):2831-7.

13. Stewart R, Morrow M, Hammond SA, Mulgrew K, Marcus D, Poon E, Watkins A, Mullins S, Chodorge M, Andrews J, et al. Identification and characterization of MEDI4736, an antagonistic anti-PD-L1 monoclonal antibody. Cancer Immunol Res. 2015;3(9):1052-62.

14. Pardoll DM. The blockade of immune checkpoints in cancer immunotherapy. Nat Rev Cancer. 2012;12(4):252-64.

15. Antonia SJ, Villegas A, Daniel D, Vicente D, Murakami S, Hui R, Yokoi T, Chiappori A, Lee KH, de Wit M, et al. Durvalumab after chemoradiotherapy in stage III non-small-cell lung cancer. N Engl J Med. 2017;377(20):1919-29.

16. Antonia SJ, Villegas A, Daniel D, Vicente D, Murakami S, Hui R, Kurata T, Chiappori A, Lee KH, de Wit M, et al. Overall survival with durvalumab after chemoradiotherapy in stage III NSCLC. N Engl J Med. 2018;379(24):2342-50. 
17. Arslan D, Bozcuk H, Gunduz S, Tural D, Tattli AM, Uysal M, Goksu SS, Bassorgun Cl, Koral L, Coskun HS, Ozdogan M, Savas B.Asian Pac J Cancer Prev. 2014;15(6):2465-72.

18. Imai $\mathrm{H}$, Wasamoto $\mathrm{S}$, Yamaguchi $\mathrm{O}$, Suzuki $\mathrm{K}$, Sugiyama $\mathrm{T}$, Uchino J, Minemura H, Osaki T, Ishii H, Umeda Y, et al. Efficacy and safety of first-line pembrolizumab monotherapy in elderly patients (aged $\geq 75$ years) with non-small cell lung cancer. J Cancer Res Clin Oncol. 2020;146(2):457-66.

19. Yamaguchi O, Kaira K, Hashimoto K, Mouri A, Miura Y, Shiono A, Nishihara F, Murayama Y, Noda SE, Kato S, et al. Radiotherapy is an independent prognostic marker of favorable prognosis in non-small cell lung cancer patients after treatment with the immune checkpoint inhibitor, nivolumab. Thorac Cancer. 2019;10(4):992-1000.

20. Reck M, Rodríguez-Abreu D, Robinson AG, Hui R, Csőszi T, Fülöp A, Gottfried M, Peled N, Tafreshi A, Cuffe S, et al. Pembrolizumab versus chemotherapy for PD-L1-positive non-small-cell lung Cancer. N Engl J Med. 2016:375(19): 1823-33.

21. Hellmann MD, Ciuleanu TE, Pluzanski A, Lee JS, Otterson GA, AudigierValette C, Minenza E, Linardou H, Burgers S, Salman P, et al. Nivolumab plus Ipilimumab in lung Cancer with a high tumor mutational burden. N Engl J Med. 2018;378(22):2093-104.

22. Kagamu H, Kitano S, Yamaguchi O, Yoshimura K, Horimoto K, Kitazawa M, Fukui K, Shiono A, Mouri A, Nishihara F, et al. CD4+ T-cell immunity in the peripheral blood correlates with response to anti-PD-1 therapy. Cancer Immunol Res. 2020;8(3):334-44.

\section{Publisher's Note}

Springer Nature remains neutral with regard to jurisdictional claims in published maps and institutional affiliations.

Ready to submit your research? Choose BMC and benefit from:

- fast, convenient online submission

- thorough peer review by experienced researchers in your field

- rapid publication on acceptance

- support for research data, including large and complex data types

- gold Open Access which fosters wider collaboration and increased citations

- maximum visibility for your research: over $100 \mathrm{M}$ website views per year

At $\mathrm{BMC}$, research is always in progress.

Learn more biomedcentral.com/submissions 